\title{
Animated Space-Filling Hierarchy Views for Security Risk Control and Visualization on Mobile Devices
}

\author{
Paul Craig ${ }^{\mathrm{a}}$, Xin Huang ${ }^{\mathrm{b}}$ \\ Department of Computer Science and Software Engineering, Xi'an Jiaotong-Liverpool University, \\ Suzhou 215123, China \\ ap.craig@xjtlu.edu.cn, bxin.huang@xjtlu.edu.cn
}

Keywords: Information Visualization, Internet of Things, Security.

\begin{abstract}
This paper looks at using a space-filling hierarchy view to control and visualize security settings on a mobile device. The view is optimized for readability and navigation with limited display space and provides a more fluid user-experience with a color-coded overview of security risks and buttons to fine tune security settings included in the same interface. The technique is compared with more traditional table and tree views in a user study where the users express a clear preference for the space-filling hierarchy view feeling. They felt that this view made for a more convenient and accessible user experience. This demonstrates the promise of information visualization to improve security interfaces for mobile devices and help solve an important problem for the future of multi-device multi-user interface development for the internet-of-things.
\end{abstract}

\section{Introduction}

The internet-of-things [1, 2] aims to increase the interconnectivity of electronic devices to support a variety of new networked applications. These include applications with great promise such as the home of the future, environmental monitoring networks and infrastructure management systems. The potential benefits of the IoT are profound. However, the benefits of the internet-of-things come with some significant challenges. Not the least of these is that the increased interconnectivity integral to an internet-of-things network increases its vulnerability to malevolent attacks where the network can be hijacked or sensitive personal data can be leaked due to inadequate security protection [3]. Internet-of-things security can, however, be problematic for a number of reasons such as the limitations of internet-of-things devices and the need for end user configuration.

One of the main problems with security in general is that users do not see it as being an integral part of the functionality of their application or system and do not want to take the time to manage security [4]. This attitude is somewhat justified when the security can be automated through better application design and user interaction is only really necessary to overcome short-comings of the design or implementation. This is not however the case when we need to assign different permissions to different users or fine-tune security settings in order to allow different devices to connect to a network as part of the application's functionality. These two actions sound like the job of an expert security administrator and not what we might consider as a normal user's job, but they could actually describe tasks as mundane as allowing your family exclusive access to a set of photos on social media or connecting a set of blue-tooth speakers to your phone. At the beginning of the twenty first century now that social media is ubiquitous and the internet-of-things is an increasing reality, a lot of the tasks previously in the domain of the expert security administrator are now the responsibility of your average user.

Other problems with security are related to interface design. Security interfaces tend to include a confusing sequence of menus and options that can be hard to navigate and comprehend for typical users. Without being an actual trained expert in security it can be difficult to find out what the different options actually mean and what their effect is on the overall state of the system. This problem is even more serious for mobile devices as different input modalities and a smaller display space can make it harder to operate menus and widgets [5]. Interaction on a mobile device also needs 
to be consistent with its natural setting and the fact that a user is more likely to be engaged in some other activity and less willing to take time to learn the interface or adapt to difficult controls. Mobile interaction should be natural, fluid and seamless [6]. This description is the complete polar opposite of a typical security interface experience.

In this paper we look at steps toward improving security interfaces by adapting them to mobile usage. More specifically, we consider adapting an information visualization [7] animated [8, 9] space filling layout [10] to support more usable security control and visualization. This layout allows us to provide an overview of a device's security status using color coding and optimizes the utility of limited screen space to make inaccurate selections less problematic. Animation is used to smooth the transition between different views whenever the user makes a selection and allows for a more flud user experience.

\section{Design}

The design of our mobile security control and risk visualization is shown in figure 1 . The user can click on different visible labeled rectangles to select different groups and subgroups of security options to navigate a hierarchy of security options. Individual security access options can be set on leaf nodes and nodes are color coded according to their security risk. Nodes are green if there is no associated security risk, dark red for a high security risk and lighter red for a lower-level security risks. When a user clicks on a leaf node they not only change the color coding of that node but also the color of all its ancestor nodes so they can view the effect of their action on the entire system and gain an overview of whole system's security.
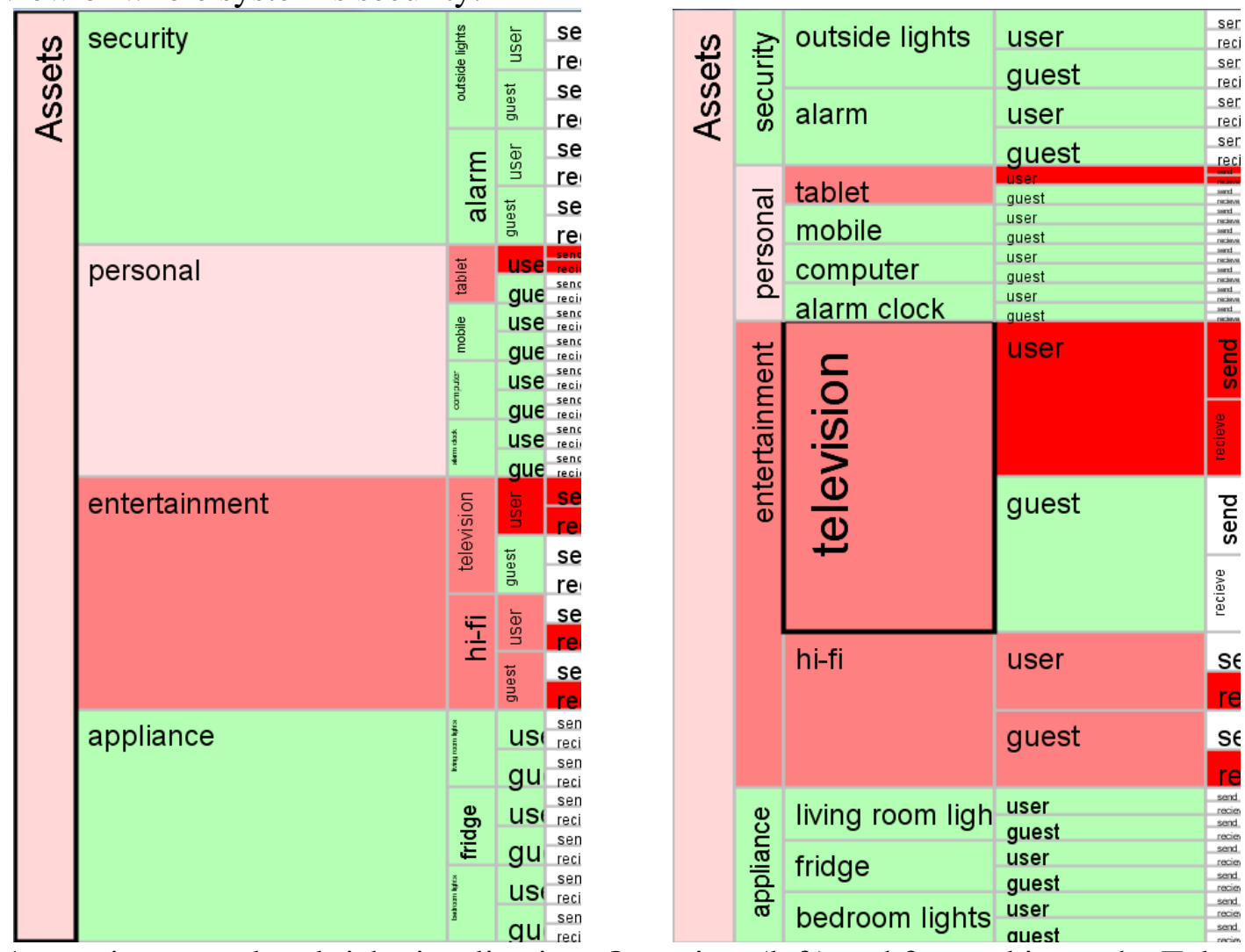

Fig. 1 security control and risk visualization. Overview (left) and focused in on the Television entertainment device node (right)

The layout of our visualization (see figure 2) is adapted from an application developed to view taxonomic hierarchies [11]. This is optimized for readability giving priority to selected nodes, their children and siblings. These nodes can also be selected to navigate down through the hierarchy and their ancestors can be selected to navigate back up the hierarchy. In either case animation is used to smooth the transition between views allowing the user to adjust easily to each new selection. 

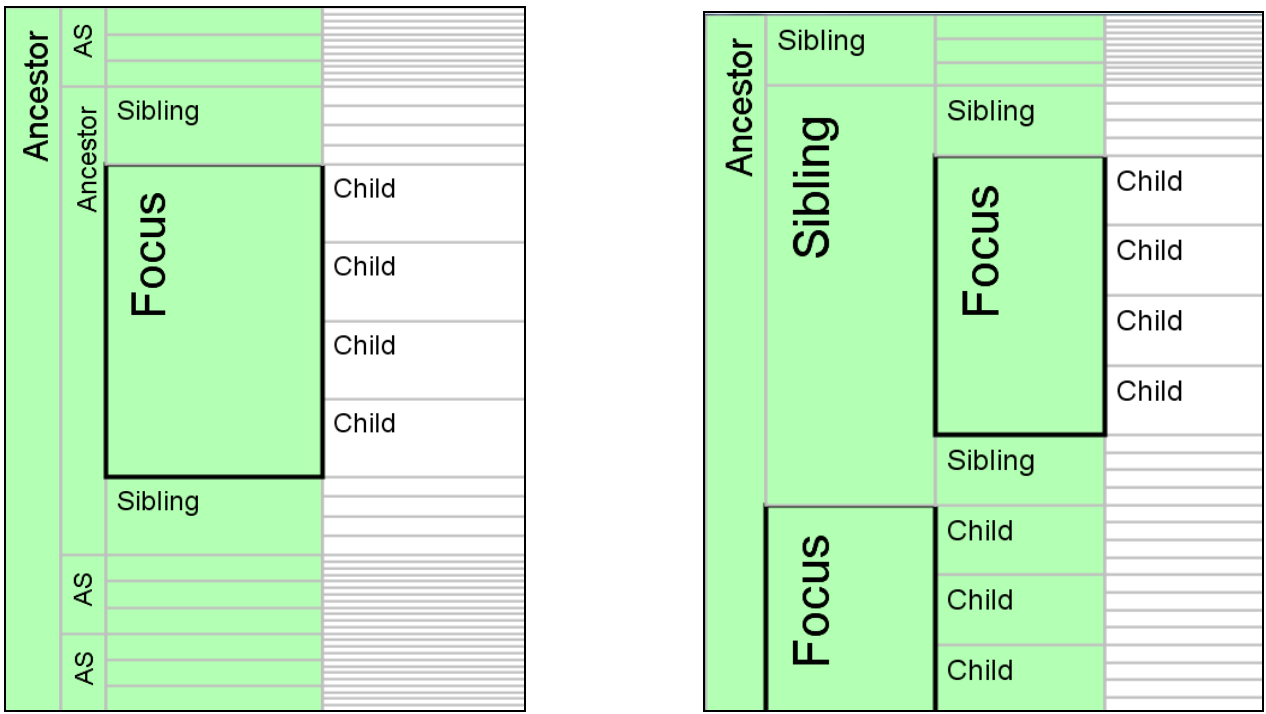

Fig. 2 Layout for one selected node (left) and two selected nodes (right). Priority vertical and horizontal spacing is given to children of the selected focus node, then siblings, then siblings of any ancestor (labelled AS). This allows the user to view interesting nodes and navigate the hierarchy.

\begin{tabular}{|l|l|l|l|}
\hline Asset & Access & Outcome & Impact \\
\hline Sensor data & Node & Disclosure & High \\
\hline & & Modification & Middle \\
\hline & Loss & Low \\
\hline & & Interruption & High \\
\hline & & Disclosure & Middle \\
\hline & & Lodification & Low \\
\hline & & Interruption & Middle \\
\hline & & Disclosure & Low \\
\hline
\end{tabular}

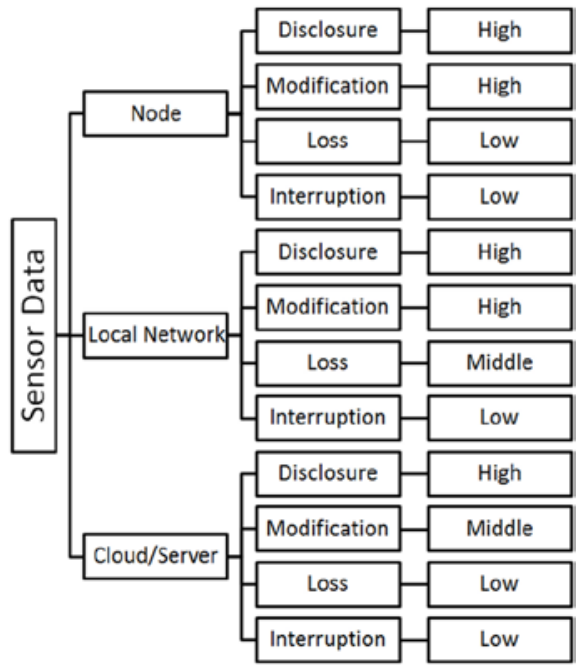

Fig. 3 a traditional table view and a standard tree view.

\section{Evaluation}

We evaluated the interface by comparing it with two alternative layouts. These were a traditional table view and a standard tree view (see figure 3). We enlisted twelve users and asked them to rate each representation out of five for usability and effectiveness. While the space filling layout achieved a low score for learnability, since the users where already familiar with the other two representations, it placed in front of the other interfaces for all other measures. The space-filling layout was marked as marginally more usable and ledgible on a desktop pc but considerably higher (above four from below three) on a mobile phone. Here users felt that the larger buttons for selecting nodes would be an advantage. The users were also impressed by the animation effect and they were happier witht the interface as they had more time to familiarise themselves with the layout. They also felt that this interface was better at handling more items on a smaller display and gave them a better overview of the overall system security. They felt like more usable interfaces like this would encorage them to be more aware of security settings and potential security risks allowing them to get more out of networked multi-device multi-user applications. 


\section{Conclusion}

We have developed and evaluated a new interface for visualizing and controlling security risks on a mobile device. The technique uses an animated space-filling layout optimized for readability and navigation, and demonstrates the potential of information visualization to improve security interfaces for mobile devices. This type of development could help solve an important problem for multi-device multi-user interface development in the future where security and user security usability are an increasingly pressing concern.

We plan to integrate this application into a security framework that helps control and manage user access in a network of interconnected devices with multiple users, and further develop the interface to incorporate more sophisticated risk analysis to differentiate between the risks from different types of attack. This should make the interface more practical and further bolster the case for usable-security and the use of information-visualization interfaces to help normal users manage security.

\section{References}

[1] Sun, Y., et al., Advances on data, information, and knowledge in the internet of things. Personal and Ubiquitous Computing, 2014. 18(8): p. 1793-1795.

[2] Weber, R.H. and R. Weber, Internet of Things. 2010: Springer.

[3] Huang, X., et al., SecIoT: A Security Framework for the Internet of Things. accepted to appear in Security and Communication Networks, 2015. 8(8).

[4] Albrechtsen, E., A qualitative study of users' view on information security. Computers \& security, 2007. 26(4): p. 276-289.

[5] Bongshin, L., et al., Beyond Mouse and Keyboard: Expanding Design Considerations for Information Visualization Interactions. Visualization and Computer Graphics, IEEE Transactions on, 2012. 18(12): p. 2689-2698.

[6] Roberts, J., et al., Visualization Beyond the Desktop-the next big thing. 2014.

[7] Card, S.K., J.D. Mackinlay, and B. Shneiderman, eds. Readings in Information Visualization: Using Vision to Think. 1 ed. The Morgan Kaufmann Series in Interactive Technologies. 1999, Morgan Kaufmann: San Francisco. 686.

[8] Craig, P., N.R. Seiler, and A.D.O. Cervantes, Animated Geo-temporal Clusters for Exploratory Search in Event Data Document Collections, in Information Visualisation (IV), 2014 18th International Conference on. 2014, IEEE. p. 157-163.

[9] Craig, P., J. Kennedy, and A. Cumming, Animated Interval Scatter-plot Views for the Exploratory Analysis of Large Scale Microarray Time-course Data. Information Visualization, 2005. 4(3): p. 149-163.

[10] Stasko, J., M. Guzdial, and K. McDonald. Evaluating Space-Filling Visualizations for Hierarchical Structures. in IEEE InfoVis, Late Breaking Hot Topics. 1999. San Francisco, California, USA: IEEE Computer Society Press.

[11] Craig, P. and J. Kennedy. Concept Relationship Editor: A visual interface to support the assertion of synonymy relationships between taxonomic classifications. in Visualization and Data Analysis 2008. 2008. San Jose, CA: Society of Photo-Optical Instrumentation Engineers, Bellingham, WA. 